\title{
Case study of virtual organization learning and knowledge testing environments
}

\author{
Kazys Baniulis \\ Computer Networking Dept., Kaunas University of Technology (KTU), \\ Associate professor, Rokishkio st. 7-1, LT-3005 Kaunas, Lithuania, \\ e-mail: kazysba@pit.ktu.It \\ Bronius Tamulynas \\ Computer Networking Dept., KTU, \\ Associate professor, Vytenu str. 65, LT-4302 Kaunas, Lithuania, \\ e-mail: bronius@pit.ktu.It \\ Nerijus Aukstakalnis \\ Computer Networking Dept., Kaunas University of Technology (KTU), Studentu 50-402, \\ e-mail: nerijus.aukstakalnis@ktu.It
}

\begin{abstract}
The proposed web-based knowledge assessment is based on flexible educational model and allows to implement adaptive control of learning process as well as to implement knowledge testing environment according to the requirements of student's knowledge level, their personal abilities and his subject learning history. The learner knowledge model can be constructed as a sub graph of the global knowledge domain graph. The paper presents the architecture of student self-evaluation and on-line assessment system TestTool. The system is explored as an assessment engine capable to support and improve the individualized intelligent self-instructional mode of learning, grounded on the GRID distributed service architecture.
\end{abstract}

Keywords: Learning environment, knowledge testing, domain of knowledge, learning objects, data structures, GRID service architecture.

\section{INTRODUCTION}

In order to promote an effective learning process it is necessary to individuate a suitable technological infrastructure able to support such process allowing each learner to use in a transparent and shared manner all the resources already existing on-line. In the innovative vision the learning process within a learning environment can be effective only using an approach which considers some fundamental characteristics of learning activity and learning within a contextualized environment $[1,2]$.

Another fundamental and innovative aspect is the possibility of personalizing the learning process with respect to the needs of each learner. Many recent studies stress the importance of the particular learning style of each student in order to foster better learning results, so we will investigate strategies and methods to determine first the learning style preferred by the student, then, on this basis, suitable methodologies which allow performing a personalized educational process, related to the specific characteristics of the learner. So, auto adaptive systems can be defined as the capability and the intelligence of learning environments which can be increased by using suitable and innovative domain of knowledge and knowledge state of learner [1, 2 and 3].

The innovative research on the GRID goes in this direction and the GRID, at the moment, seems to be the technological infrastructure that fits such requirements best. We are trying to realize this conception by using TestTool (TT) knowledge testing environment $[5,6]$.

\section{VIRTUALITY OF LEARNING ENVIRONMENTS}


When the learner enters the simulated environment, he finds himself in some initial state, and his objective is to move to some final (solution) state by performing appropriate actions, operations and moves. From the cognitive perspective, when the learner tackles a new problem, he uses a number of cognitive strategies that involve, among others, inductive and deductive reasoning, learning by trials and errors and insight. The user performs a sequence of (either mental or concrete) operations that allow him to move from one state to another until the final (solution) state of the problem is reached. So, simpler terms this principle may be defined as mapping from the knowledge state of a student to the domain of knowledge space of the problem [1].

According to the definitions presented in the FR6 IST programme IP project ELeGI a domain of knowledge is a collection of items (e.g., problems, questions, exercises, examples, and other learning objects (LO)) in a given field of knowledge. The knowledge state of a learner is the set of all items this student actually masters. In the domain of knowledge the items are linked by surmise relation, which allows identifying the prerequisites for each item. This relation is a partial order for representing implication relations among items in a given domain of knowledge. The knowledge state of the learner is mapped into a latent skill state, and the mapping itself is called skills map. The knowledge space is just a model of the cognitive organization of some learning material. As such, to become a valid representation with respect to some existing population of learners, it has to be tested empirically. The learner knowledge model [2, 3] can be constructed as a sub graph of the global knowledge domain graph. Such knowledge representation model belongs to a most general theory concerning multi-graphs. By decomposing information into atomic units and finding the connections among the units themselves: motivational or historical type, difficulty degrees the domain knowledge model can be based on graph paradigm. The structure, therefore, consists of both the information units and their links.

Such way of organization training environments enables tracking the improvements of the user along the whole training process. This implies a dynamic adaptation of the system to the user's skills and performance (personalization) so that his motivation and mental activity remains at a rational level during the whole training session. The mechanism at the basis of this adaptation is performance and skill assessment and monitoring. The skill is the capability to perform a given sequence of operations in a purposeful way and the meta-skill is ability to combine such sequences. Such considerations suggest that the tool sharing among all the users is the key element to strengthen the effectiveness of the learning process in the situated learning framework, so all the learners, everywhere and at any time, are provided with the same equipment to know and be able actively take part in the common knowledge building.

Finally, it is important for the students to use particular resources which, due to their specific character, can be present only on remote systems. In this case the learner can invoke any application from a remote system, use the system best suited for executing that particular application, access data securely and consistently from remote sites, exploit multiple systems to complete complex tasks in an economical manner, or use multiple systems to solve large problems that exceed the capacity of a single one.

\section{A CASE STUDY: DATA STRUCTURE COURSE MODEL AND STUDENT MODEL}

A case study is based on the use of graphical assessment system TestTool [5] in the Data structure study module. Course model creation. Course model can be defined as a collection of items in a given field of knowledge. Course structure is created according to learner's needs, their aims and objectives. Hierarchical principle is employed to organize the learning context, i.e. it can be enumerated or rendered graph using relational topics which are studied in any order. Various ways of analysis and graphical rendering are employed to aggregate course structure. One of the many possible forms is the concept map. You should begin with the name of the key topic or concept and link it to a number of related concepts. Then write in the names of concepts related to these and connect them too. Another technique for content presentation can be used: hierarchical lists, chronological lists, flow charts, algorithmic schemes, content matrices, causal schemes, etc. 


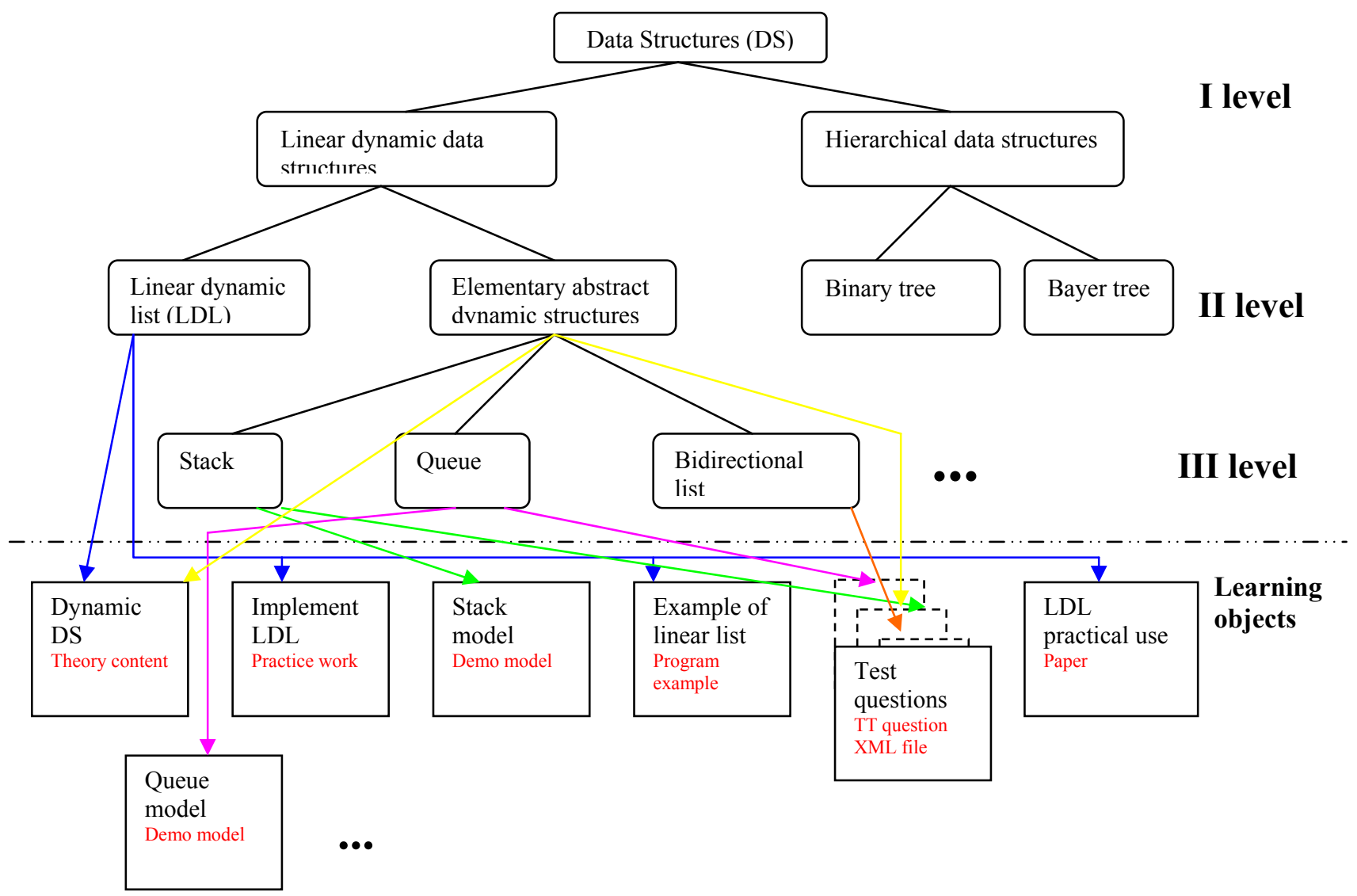

FIGURE 1: Data structure course model

A case study: Teachers-experts define what learners should know according to the three levels of knowledge (basic, intermediate and expert). These definitions may be used to develop course model and to form student model sub sets. Also criteria of educational goals according to Bloom's taxonomy can be used, when creating models $[2,7]$. Course model creation should be very simple:

$\checkmark$ the author creates learning objects and describes their attributes;

$\checkmark$ teacher-expert defines relations between one LO and a couple of other LO possessing some kind relationships;

$\checkmark \quad$ there can be a couple of different relationships between two LO;

$\checkmark$ possible types of relationship are defined initially or added/modified later.

Attributes and relationships (LO metadata) are described in XML, according to existing e-learning standards. In the same way as TestTool test-exercises (LO data) are described.

The main reasons to use TestTool basis to realize the first domain of knowledge are:

$\checkmark$ it is authorized environment and could be easy modified according to our needs;

$\checkmark \quad$ it is convenient to use XML files for LO presentation adding only several needed new features.

Fig. 1 shows domain of knowledge as data structure course model and student model, as a subset of Course model. According to various criteria subsets of Course Semantic Network are formed to aggregate student model. Graph analysis methods take into account learner possibilities and his learning progress.

\section{GRID DISTRIBUTED SERVICE ARCHITECTURE AND INTELLIGENT LEARNING}

Naturally, we often must answer the question: should the conventional web-based technologies transferred directly onto the GRID, or there should be a more complex domain and more complex architecture? Evidently, there is an abundance of e-learning practical and pilot cases which have been realized with conventional web-based technologies and there are no obvious reasons to transfer them directly onto the GRID. Learning subjects typical of science adjoined and tied to science and research, experienced learning with learners' involvement into more complex domains and more complex e-learning application architectures consistent with Grid distributed service oriented architecture should prevail. 
Looking at the integrity and interoperability of distributed learning object systems the experimental TestTool version for GRID was implemented [6]. The following steps to transform the existing TestTool system to GRIDTT version are needed:

1. TestTool system is created as distributed system based on Web services. Three large subsystems of services are created: Client, LO Repository closely linked with LO Registry, Learner Repository. Client is a general subsystem which realizes interfaces for TT users. LO repository is the service for storage, registration and retrieval of LOs. Learners' repository is a learners' database containing individual student data records and testing results. The typical service sequence for learner is:

- Student logs in through Learner Repository and chooses an eligible test from the given list;

- LO Registry is searched for the chosen test LO, LO Repository address and LO identification data are retrieved;

- Learner Repository calls LO Factory and creates instances of aggregated questions;

- A learner communicates with the visualized LO to answer the question;

- LO sends the answer and its evaluation to the Learner Repository.

2. Course model and Learner model solutions are performed in order to fulfil knowledge domain requirements (Figure 2).

There are a lot of additional tools in the existing integrated systems. Creation of Web service based e-learning system produce new challenges. Due to more complex architecture, course administration becomes more complicated too. Administrator has to aggregate the course from learning objects distributed in various places. Course aggregation becomes complicated to inexperienced user and possibility is high that something will be missed or mistakes will be made. One of the main goals of the distributed system (learning GRID) is to create and improve services to increase the level of automation and users utility.

High level visualization for course administration reduces interest in the structure of the course itself and administrator has to specify LO and relations between them. Graphical course aggregation tool or course visualization using tree structures in HTML file are any useful. LO is then located by the system of queries according to the attributes administrator has specified.

Users aren't happy when they have to install additional software for the system to work. To solve this problem technologies supporting standard operating system should be used. Currently, the new version of TT based on Web services is being created. Tests, created using earlier version of TT Author program, will be usable in the new version, too. XML converter was created in order to convert questions into new format. In this case reusability of context is secured. The XML converter itself can be used to complete standardizing learning contexts.

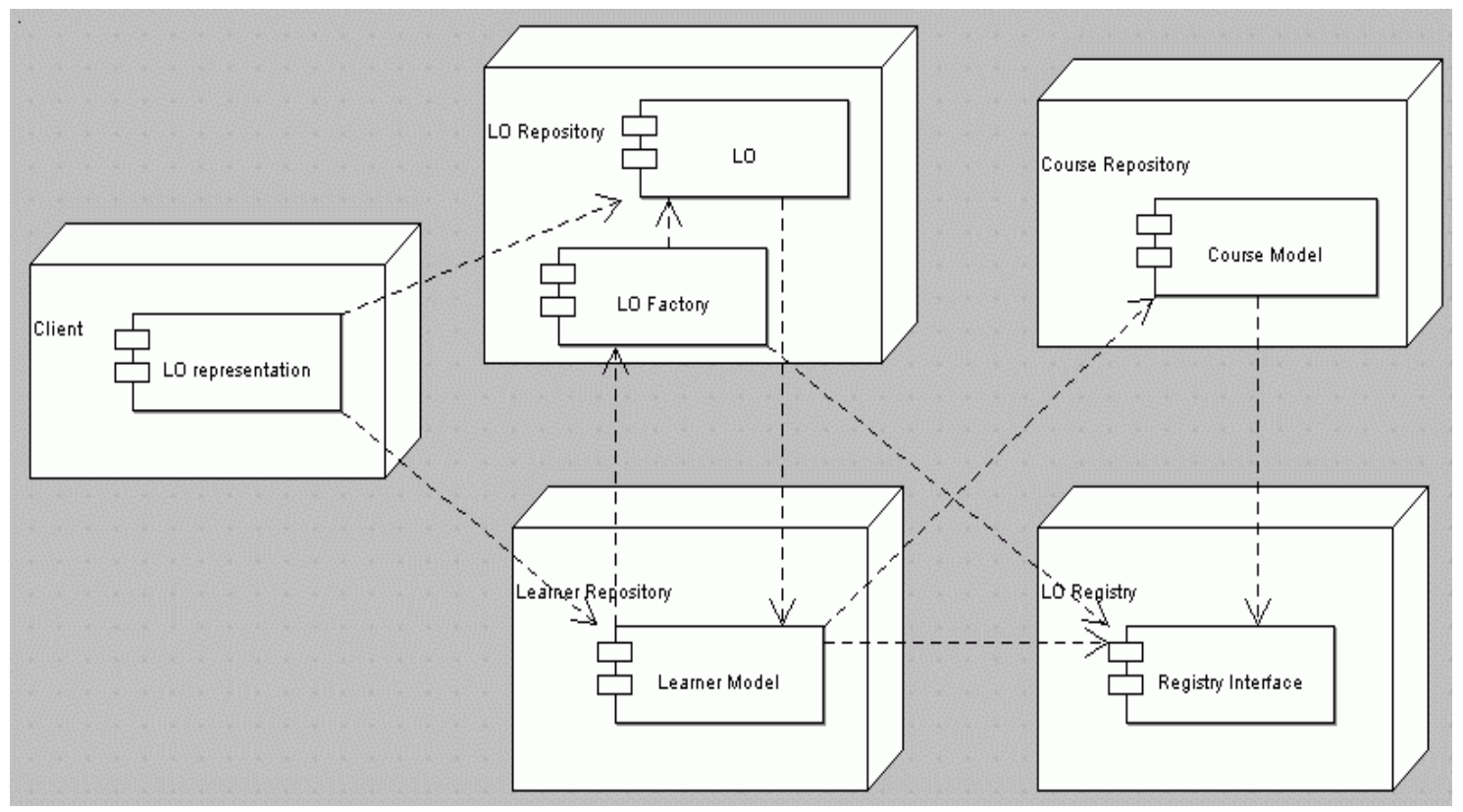

FIGURE 2: Conceptual learning environment using GRID service architecture 


\section{META DESCRIPTION OF LEARNING OBJECTS}

Semantic network of Course model in the new version will be used. Metadata of LO must be defined. Learning objects will be used to produce Course model, so attributes of LO should be described in metadata. Metadata will be described according to existing e-learning standards (IMS Meta-data and IEEE LOM). In the standard metadata, all elements, describing LO, are divided into 9 groups. At the moment, most useful are:

1. General - Information Groups describing learning object as a whole;

2. Educational - Educational or pedagogic features of the learning object;

3. Relation - Features of the resource in relationship to other learning objects.

4.

Most important elements in General group are: Identifier - globally unique label for learning object; Title - learning object's name; Language - learning object's language (or Language without Country sub code - it implies intended language of the target audience. "None" is also acceptable); Description - describes learning object's content; Keyword - contains keyword description of the resource; Structure - underlying organizational structure of the resource; Aggregation level - the functional size of the resource. The level is from 1 to 4 . Level 1 means the smallest level of aggregation, e.g. raw media data or fragments. Level 2 refers to a collection of atoms, e.g. an HTML document with some embedded pictures or a lesson. Level 3 indicates a collection of level 1 resource, e.g. a 'web' of HTML documents, with an index page that links the pages together or a unit. Finally, level 4 refers to the largest level of granularity, e.g. a course.

Elements in Educational group: Interactivity type - the type of interactivity supported by the learning object; Learning resource type - specific or most dominant kind of resource; Interactivity level - level of interactivity between the end user and the learning object; Difficulty - difficulty to work through the learning object for the typical target audience; Typical learning time - approximate or typical time it takes to work with the resource.

Elements in Relation group: Kind - nature of the relationship between the resource being described and the one identified by Resource. Types according to Dublin Core: ispartof, haspart, isversionof, hasversion, isformatof, hasformat, referentes, isreferencedby, isbasedon, isbasisfor, requires, isrequiredby;

\section{An example of main elements in Metadata description of learning objects in Data structure course}

Structure: Possible values: Aggregate, Linear.

Learning Resource type: TestTool question XML file, practice work, paper, theory content, demo model, program example.

Keywords:

Type of learning content: content, algorithm, programming.

Programming language: Java, $\mathrm{C}++$, Pascal

Difficulty: minimal, intermediate, expert.

Relation Kind:

ispartof, haspart example: Stack - loop;

isbasedon, isbasisfor example: Content - Algorithm; Algorithm - Program. 


\section{An example of LO description in XML}

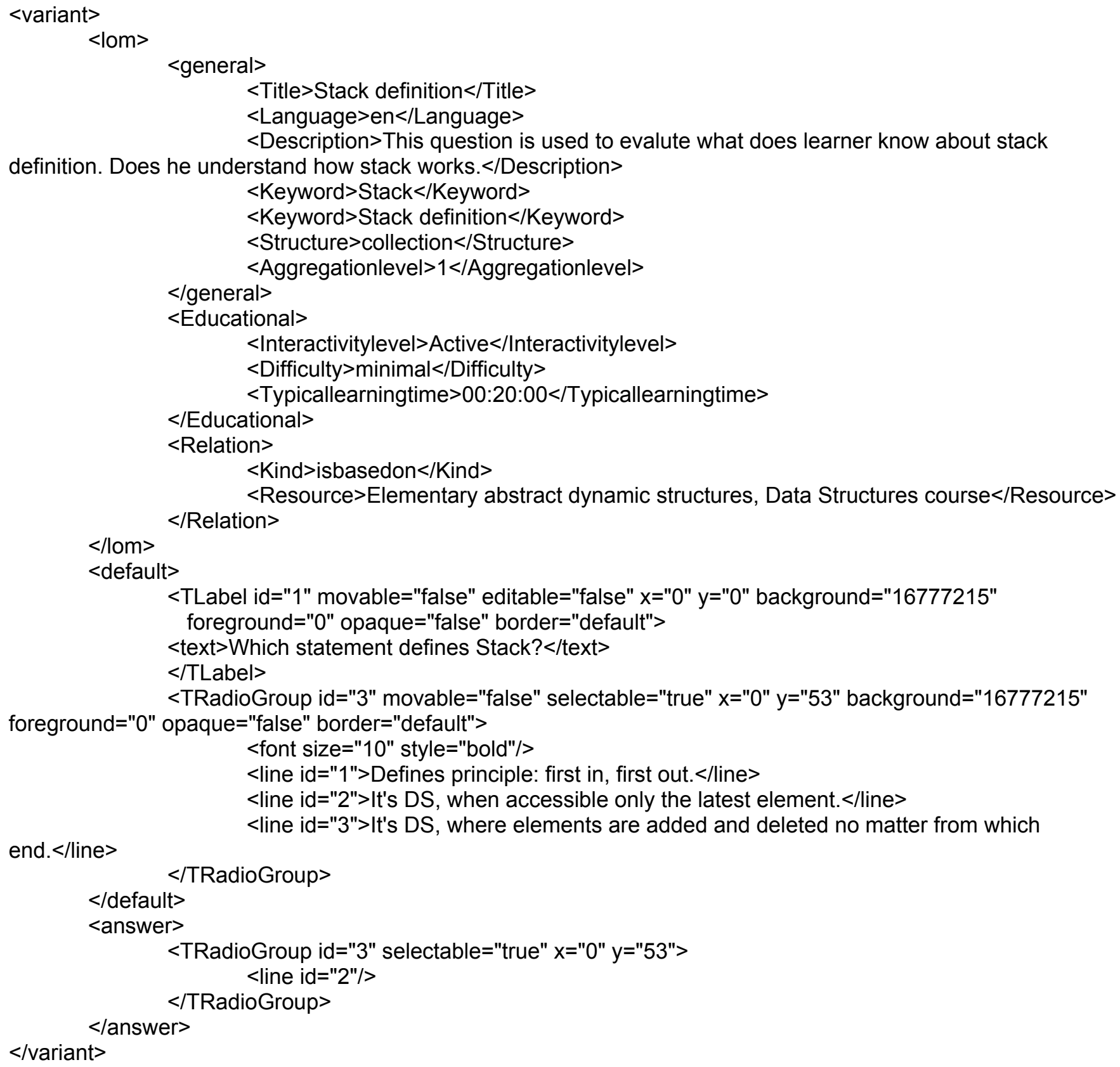

\section{CONCLUSIONS AND DISCUSSIONS}

Our research consist of two parts: conceptualization of virtual organization of learning and knowledge testing environments and development of web-services based knowledge assessment TestTool version. In the next stage there was created experimental service-oriented TestTool 1.0 version which implements GRID environment based on Globus toolkit. Globus tools are used for accumulation of distributed resources into one integrated system, but not for distribution of data and tasks to available computing resources. Such implementation has higher security level and usability of distributed learning resources. All web services embedded in TT Grid aware implementation are corresponding to the Grid requirements (actualizing concrete interfaces and using particular protocols of communication). While student is working with test, couple of temporary LO grid services are created and combined. These services are the most dynamical part of TestTool. When test is composed, selection of services is performed according to meantime accessible LO services (selection is performed using LO registry). All needed services according to metadata may be created on closer or less laden server. That's similar to reuse software components: at the runtime, when test execution or course content is needed. We suppose that all components already are created. 
It is needed to split parts which are not closely relative in order to make TT as Grid oriented implementation in distributed E-LeGI infrastructure with multiple LO repositories. Also there should be described interfaces for communication between these parts and later suitable interfaces should be implemented using Globus distributed systems creation possibilities.

\section{REFERENCES}

[1] Capuano, N., Gaeta, A., Laria, G., Orciuoli, F., Ritrovato, P. How to use technology for building next generation learning environments. Proceedings of the 2nd International LeGE-WG Workshop: Towards a European Learning GRID Infrastructure. 3-4 March, 2003, Paris, France.

[2] Baniulis K., Tamulynas B. Intelligent support of web-based knowledge assessment system. IEEE International Conference on Advanced Learning Technologies proceedings, 9-12 September 2002, Kazan, Tatarstan, Russia.

[3] Baniulis K., Tamulynas B. Flexible Learning in an Intelligent Tutoring Environment // In the book: New Media and Telematic Technologies for Education in Eastern European Countries,1997, Twente University Press, p.p. 395-409.

[4] Reklaitis, V., K. Baniulis, Okamoto, T. Shaping e_Learn applications for a service oriented Grid. Proceedings of the 2nd International LeGE-WG Workshop on e-Learning and Grid Technologies: A Fundamental Challenge for Europe. 3-4 March, 2003, Paris, France.

[5] Baniulis, K., Reklaitis, V. TestTool: Web-based Testing, Assessment, Learning. Informatics in Education, ISSN 1648-5831, Volume 1, 2002, Vilnius, Institute of Mathematics and Informatics, p.p. 17-30.

[6] Reklaitis, V., Baniulis, K., Paulikas, G. Implementation of Assessment Web-service for TestTool. Proceedings of the International Conference "Advanced learning technologies and applications (ALTA'03)', Kaunas, 11-12 September, 2003, Lithuania, p.p. 39-40.

[7] Anderson, L. and Krathwohl, D. A Taxonomy (2001) for Learning, Teaching and Assessing: A Revision of Bloom's Taxonomy of Educational Objectives. Longman, New York. 